\title{
A Low-Complexity and Efficient Encoder Block Mode Decision for Distributed Residual Video Coding
}

\author{
Chunyun $\mathrm{Hu}^{1,2}$, Binjie $\mathrm{Hu}^{1}$ and Yunhui Xiong ${ }^{3 *}$ \\ ${ }^{1}$ School of Electronic and Information Engineering, South China University of \\ Technology, Guangzhou, China \\ ${ }^{2}$ College of Electronic and Engineering, South China Agricultural University, \\ Guangzhou, China \\ ${ }^{3}$ College of Science, South China University of Technology, Guangzhou, China \\ hcy2182@scau.edu.cn,eebjiehu@scut.edu.cn,yhxiong@scut.edu,cn
}

\begin{abstract}
Existing encoder block mode decision (EBMD) algorithms often bring computation load and complexity at the encoder. In the distributed residual video coding (DRVC) system, based on the analysis of the statistical characteristics of the decoder side information (DSI), a simple scheme called EBMD-DRVC is introduced to maintain the low-complexity nature of the encoder. EBMD-DRVC only employs the value of the residual pixel at the encoder to classify the macro blocks into Intra mode, Skip mode and WZ mode. If a Skip mode block cannot be replaced by the co-located block in the DSI because of its unsatisfied quality, the decoder can improve the quality. The simulation results show that EBMD-DRVC is efficient in mode decision and has competitive rate distortion performance when compared with DISCOVER system. Due to having a lowcomplexity encoder, EBMD-DRVC is promising in practice.
\end{abstract}

Keywords: Distributed residual video coding; Encoder block mode decision; lowcomplexity encoder

\section{Introduction}

Distributed Video Coding (DVC) is an alternative video coding scheme, which is famous for its simple encoder architecture and is more suitable for the applications such as wireless digital video cameras, low-power video sensor networks, video surveillance cameras and so on. The theoretical foundations of DVC are the Slepian-Wolf theory which is about the lossless distributed coding and Wyner-Ziv theory which is about the loss distributed coding. These theories suggest that the statistical redundancies in a (video) signal can be exploited at the decoder side with only a limited performance loss with respect to a system that exploits the redundancies at the encoder. This facilitates the design of a simple video encoder at the cost of the increased complexity at the decoder because of the motion-compensated prediction shifting from the encoder to the decoder. The well-known DVC architectures have been developed by researchers in Stanford University, mainly including pixel-domain DVC (PDDVC) [1], transform-domain DVC (TDDVC) [2] and distributed residual video coding (DRVC) [3].

During the past decade, DVC has been popularly studied. The research hotspots mainly focus on improving the coding efficiency, decreasing the system latency and removal of the feedback channel. In order to improve the coding efficiency, well-known strategies including side information refinement [4-5], more accurate correlation noise model [6], more effective reconstruction [7] and block mode decisions (BMD) [8-16] have been proposed. In order to decrease the latency, low-delay DVC systems based on motion-

${ }^{*}$ Corresponding Author 
compensated extrapolation [17] and DVC systems using entropy coding without iterative channel codes have been introduced in [18]. In order to remove the feedback channel, rate estimations at the encoder have been presented.

We studied the problem of BMD and knew that BMD algorithms can be implemented at the encoder (defined as encoder BMD, EBMD)[8-14], or at the decoder (defined as decoder BMD, DBMD)[15-16]. DBMD is more effective than EBMD because the accurate side information (SI) and correlation noise model can be obtained at the decoder. But DBMD requires a feedback channel to send the mode information to the encoder which brings the latency. On the contrary, EBMD suits for the scenario without the feedback channel and has less latency which is more practical than DBMD. But the existing EBMD often brings computation load and complexity at the encoder.

This paper focuses on EBMD in DRVC system. Since the encoder is limited in power, memory and computational capability, a very simple and efficient scheme called EBMDDRVC is introduced to maintain the low-complexity nature of the encoder. The proposed EBMD-DRVC has two advantages. One is that the mode decision criterion is very simple which only employs the value of the residual pixel at the encoder to determine the block mode without any heavy computation. The other is that the threshold is fixed.

The remainder of this paper is organized as follows. Section 2 reviews the existing EBMD algorithms. Our EBMD-DRVC scheme is proposed in detail in Section 3. In Section 4, experimental results are shown and discussed. Finally, we conclude the paper in Section 5.

\section{Review of the Existing EBMD Algorithms}

In the existing EBMD algorithms, Intra mode and WZ mode are often introduced. In literature [8-9], the mode selection depends on the sum of absolute differences (SAD) between the blocks as an indication of the temporal coherence. If the SAD is less than the threshold value, WZ mode is chosen, or else Intra mode is chosen. In [10], both spatial and temporal block characteristics are taken into consideration by calculating the pixel variance of each block and the SAD, respectively. In [12], an iterative algorithm is proposed to dynamically select either Intra mode or WZ mode to encode a DCT block. In order to make more accurate mode decision, SI is required at the encoder. In literature [13], a cost function composed of compression rate and distortion is calculated, and the block mode with the minimum cost is chosen. In addition to Intra mode and WZ mode, skip mode used in [14] is also introduced which can save the transmission data and therefore improve the RD performance.

Although all the above EBMD algorithms can improve the coding efficiency, they undoubtedly bring computation load and complexity at the encoder due to the computation of the metrics such as SAD, compression rate, distortion function and so on. Furthermore, if there are some thresholds which should be pre-defined, the users do not have a clue how to set them. Those disadvantages prevent EBMD from being widely used in real-world applications. In order to make EBMD more practical, we propose the EBMD-DRVC scheme to solve these problems effectively.

\section{Proposed EBMD-DRVC Scheme}

Based on the analysis of the statistical characteristics of the DSI, a simple scheme called EBMD-DRVC which only employs the value of the residual pixel at the encoder to classify the mode is introduced. 


\subsection{System Diagram of EBMD-DRVC}

Figure 1 illustrates the EBMD-DRVC architecture proposed in this paper. Set that the whole video sequence is divided into WZ frame and KEY frame by GOP $=2$ where $X_{2 k}$ is WZ frame and $X_{2 k+1}$ is KEY frame encoded by H.264/AVC Intra. The reference frame is defined as

$$
X_{r e}=\left(\hat{X}_{2 k-1}+\hat{X}_{2 k+1}\right) / 2
$$

where $\hat{X}_{2 k-1}$ and $\hat{X}_{2 k+1}$ are KEY frames decoded by H.264/AVC Intra. The residual frames at the encoder and decoder are defined as (2) and (3), respectively

$$
\begin{gathered}
R=X_{2 k}-X_{r e} \\
R^{\prime}=Y_{2 k}-X_{r e}
\end{gathered}
$$

where $Y_{2 k}$ estimated by MCFI [19] is the SI of $X_{2 k}$ and is computed using (4) where $m v, m v=\left(m v_{x}, m v_{y}\right)$, is the estimated motion vector.

$$
Y_{2 k}=\frac{1}{2}\left[\widehat{X}_{2 k-1}\left(x+m v_{x}, y+m v_{y}\right)+\widehat{X}_{2 k+1}\left(x-m v_{x}, y-m v_{y}\right)\right]
$$

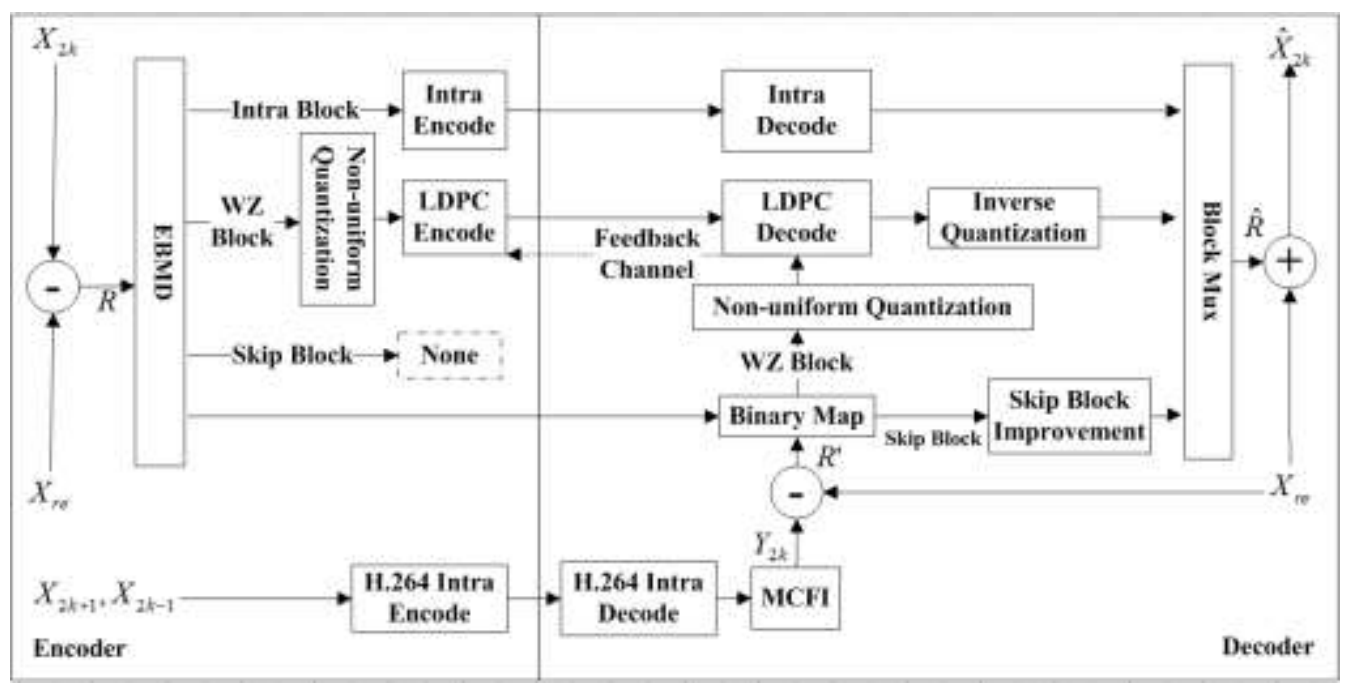

Figure 1. System Diagram of EBMD-DRVC

At the encoder, $\mathrm{R}$ is divided into non-overlapping $4 \times 4$ macro blocks. Each block is classified into Intra mode, Skip mode or WZ mode using the EBMD module and a binary mode decision map employing variable length coding (VLC) is sent to the decoder. For Intra blocks, the Intra codec is used (including DCT, scalar quantization and VLC). For WZ blocks, non-uniform quantization and LDPC code are used. For Skip blocks, they are skipped without transmission.

At the decoder, Intra blocks are intra decoded. WZ blocks are LDPC decoded and inversely quantized. Skip blocks are replaced by the co-located blocks in $R^{\prime}$. If the quality of the co-located blocks is not good enough, the decoder can improved the quality. According to the binary decision map, all the blocks will combine to get $\hat{R}$, and finally $\hat{X}_{2 k}=\hat{R}+X_{r e}$ 


\subsection{Analysis of the Decoder SI (DSI) $R^{\prime}$ and the Quantization Index $R_{q}^{\prime}$}

In DRVC system, $R^{\prime}$ is regarded as the SI of $R$. We focus on the statistical characteristic of $R^{\prime}$. The probability distribution curves of the residual pixels in any $R^{\prime}$ of Hall Monitor, Foreman, Coastguard, and Soccer videos are illustrated in Figure 2. It shows that each curve is sharp near 0 which means that the pixel value is centered on 0 .

It is because we can regard $R^{\prime}$ as the motion-compensated errors between the adjacent video frames by comparing (1), (3), and (4). Since most backgrounds and foreground in the adjacent frames are changed a little, the motion-compensated errors for these regions are very small and that results in the case $R^{\prime}=Y_{2 k}-X_{r e} \approx 0$ being in the majority.

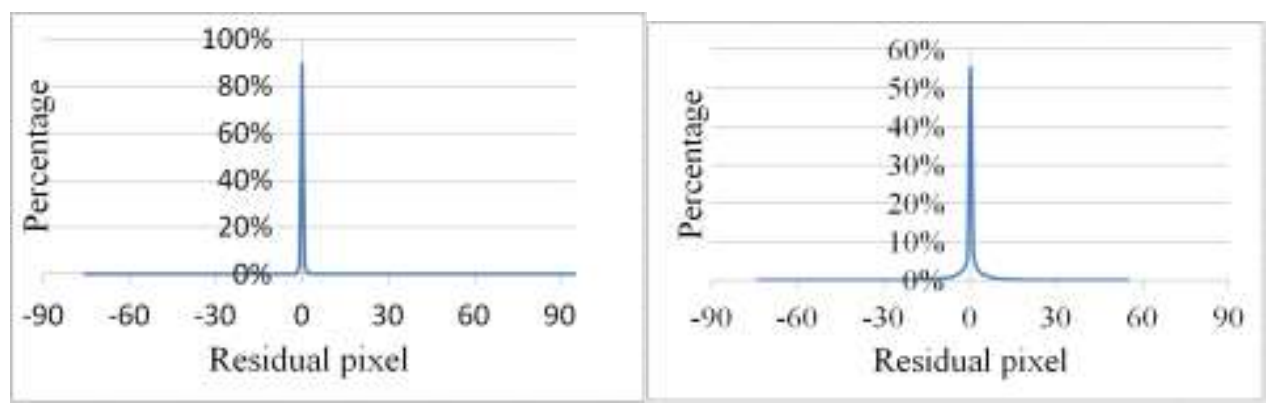

(a)

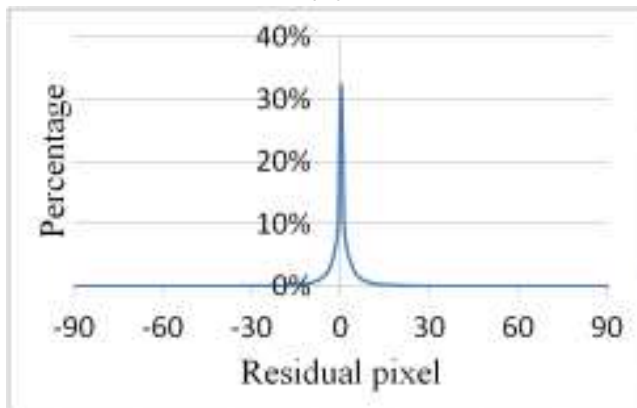

(c) (b)

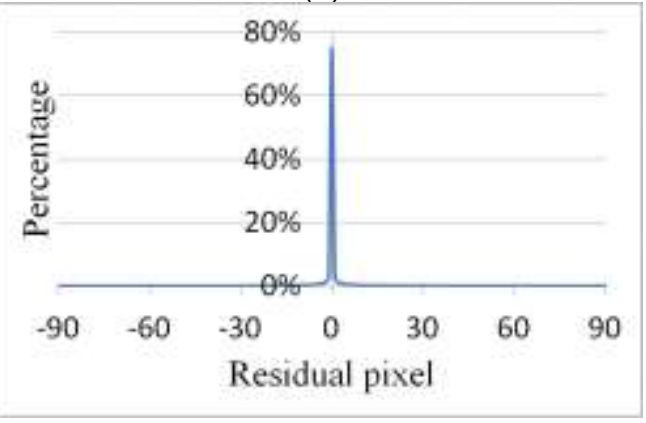

(d)

Figure 2. Probability Distribution of (a) the 13th Residual Frame of Hall Monitor, (b) the 32th Residual Frame of Foreman, (c) the 4th Residual Frame of Coastguard, and (d) the 19th Residual Frame of Soccer

Specific to the statistic characteristic of $R^{\prime}$, we employ non-uniform quantization. Set that the quantization intervals are $[-255,-31][-30,30][31,255]$ where the threshold 30 is empirically obtained and the corresponding quantization indexes $R_{q}^{\prime}$ shall be $-1,0,1$. Table 1 gives the quantization results of $R^{\prime}$ which is shown in Figure2 and we can see from it that since the case $R^{\prime}=Y_{2 k}-X_{r e} \approx 0$ is in the majority, the residual pixels falling in the interval $R_{q}^{\prime}=0$ account for more than $97 \%$, even up to $99 \%$. Specific to such characteristic, we can assume that $R_{q}^{\prime}=0$ accounts for $100 \%$. 
Table 1. Percentage of Each ${ }^{\prime}$ in $R^{\prime}$

\begin{tabular}{|l|l|l|l|}
\hline quantization interval & {$[-255,-31]$} & {$[-30,30]$} & {$[31,255]$} \\
\hline quantization index $R_{q}^{\prime}$ & -1 & 0 & 1 \\
\hline Hall Monitor (the 13th residual frame) & $0.295928 \%$ & $99.45155 \%$ & $0.252525 \%$ \\
\hline Foreman(the 32th residual frame) & $0.323548 \%$ & $99.08854 \%$ & $0.58791 \%$ \\
\hline Coastguard(the 4th residual frame) & $0.591856 \%$ & $98.78078 \%$ & $0.627367 \%$ \\
\hline Soccer(the 19th residual frame) & $0.994318 \%$ & $97.16304 \%$ & $1.842645 \%$ \\
\hline
\end{tabular}

Here the statistic characteristic of DSI $R^{\prime}$ can be summarized as follows. The value of the residual pixel concentrates near 0 and after implementing non-uniform quantization, we assume that $R_{q}^{\prime}=0$ accounts for $100 \%$.

\subsection{Proposed Block Mode Decision Criterion}

Based on the hypothesis that $R_{q}^{\prime}=0$ accounts for $100 \%$, a simple and effective block mode decision criterion is proposed. The definitions and decision criterion are:

- The size of macro block is $4 \times 4$ and there are altogether 16 residual pixels.

- The macro blocks in $R$ and $R^{\prime}$ are represented as $R_{b l o c k}$ 和 $R_{b l o c k}^{\prime}$ respectively. $\operatorname{PSNR}\left(R_{\text {block }}, R_{\text {block }}^{\prime}\right)$ is the peak signal to noise ratio (PSNR) of the macro block.

- $p_{i}$ and $p_{i}^{\prime}$ denote the residual pixel in $R_{\text {block and }} R_{\text {block }}^{\prime}$ respectively, where $p_{i}, p_{i}^{\prime} \in[-255,255] i=1,2,3, \ldots, 16$.

- Intra block: It refers to the block whose correlation with the co-located block in DSI is weak. For this kind of block, using Intra codec is more effective than Wyner-Ziv codec. Given the specific hypothesis of $R_{q}^{\prime}=0$, the case $\left|R_{q}\right|=1$ means that the correlation is weak. Therefore, A block with at least six $p_{i}$ satisfying $\left|p_{i}\right|>30$ is decided as Intra block.

- Skip block: It refers to the block whose correlation with the co-located block in DSI is strong. It can replace by the side information block. Given the specific hypothesis of $R_{q}^{\prime}=0$, the case $R_{q}=0$ means the strong correlation. In order to obtain higher $\operatorname{PSNR}\left(R_{\text {block }}, R_{\text {block }}^{\prime}\right)$, A block with all the ${ }^{p_{i} \text { satisfying }}\left|p_{i}\right| \leq 10$ is decided as Skip block.

- WZ block: A block which is neither a Intra block nor a skip block is decided as WZ block.

It can be seen that EBMD-DRVC is very simple which just depends on the value of the residual pixel at the encoder to decide the block mode without any computation of the metrics such as SAD, compression rate, distortion function and so on which are mentioned in the existing EBMD algorithms. Figure 3(a) and 3(b) show the $1^{\text {th }}$ residual frame of Foreman and the three kinds of blocks in it, respectively. 


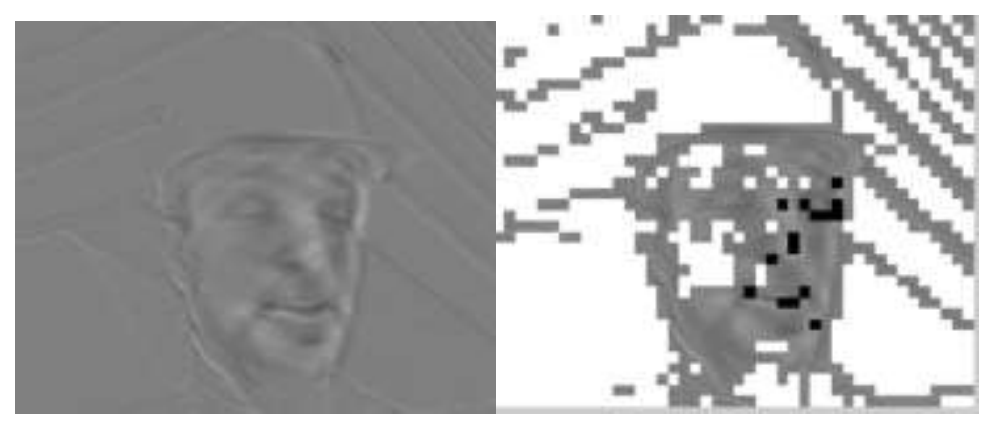

(a)

(b)

Figure 3. Display of (a) The 1th Residual Frame of Foreman, (b) The Three Kinds of Blocks which Use White, Black, and Gray to Represent Skip Blocks, Intra Blocks, and WZ Blocks, Respectively

\subsection{Improve the Quality of ${ }_{\text {block }}^{\prime}$ for Skip Block}

EBMD-DRVC is based on the hypothesis that $R_{q}^{\prime}=0$ accounts for $100 \%$ which is not always true. In reality the case $R_{q}^{\prime} \neq 0$ seldom occurs, but when it occurs that may result in wrong decisions. If a $R_{b l o c k}$ is wrongly classified as an Intra block, it will be reconstructed correctly after Intra decoding. If a $R_{\text {block }}$ is wrongly classified as a Skip block, it will be unable to be replaced by the co-located $R_{\text {block }}^{\prime}$. Because in that case, $R_{q}=0$ while $R_{q}^{\prime} \neq 0$, the correlation of the $R_{\text {block }}$ and $R_{\text {block }}^{\prime}$ is not good enough. To solve this problem, (5) can be used to improve the quality of the side information block $R_{\text {block }}^{\prime}$. The improved $R_{\text {block }}^{\prime}$ satisfies that all the $p_{i}^{\prime}{ }_{\text {in }} R_{\text {block }}^{\prime}$ are less than or equals 10.

$$
p_{i}^{\prime}=\left\{\begin{array}{cc}
10 & p_{i}^{\prime}>10 \\
-10 & p_{i}^{\prime}<-10 \\
p_{i}^{\prime} & \left|p_{i}^{\prime}\right| \leq 10
\end{array} \quad p_{i}^{\prime} \in R_{\text {block }}^{\prime}\right.
$$

\section{Experimental Results and Analysis}

Four test video sequences namely Hall Monitor, Foreman, Coastguard and Soccer with QCIF resolution at $15 \mathrm{~Hz}$ are used for the experiments. The GOP is 2. Odd frame is the KEY frame encoded with H.264/AVC Intra codec for the QP parameter equal to 16, 18, $20,24,27,30,32$ and 34 respectively. Even frame is the WZ frame. The reference frame and residual frame are the same as those described in the introduction section of the EBMD-DRVC architecture. For WZ blocks, the non-uniform quantization mentioned in Section 3.2 is used and the code length of LDPC is 396.

\subsection{Efficiency of EBMD}

Figure 4 shows the percentage of each mode in four test sequences where the QP is 24 . It shows Skip blocks in Hall Monitor account for the largest proportion, reaching 93.66\%. Intra blocks in Soccer accounting for $12.73 \%$ are more than those in other three videos. 
That means the lower the motion is, the more percentage the skip blocks account for and the higher the motion is, the more percentage the Intra blocks account for. Figure 5 shows the average PSNR (APSNR) for each kind of block which is shown in Figure 4. It can be seen that for Intra blocks, the APSNR is mainly $14 \mathrm{~dB}-17 \mathrm{~dB}$ that means the correlation between the Intra blocks and their side information blocks is weak and the Intra codec is appropriate. For WZ blocks, the APSNR is mainly $29 \mathrm{~dB}$ that means the quality of their side information blocks is medium and WZ codec is appropriate. For Skip blocks, the APSNR is as high as $43.19 \mathrm{~dB}$, usually over $39 \mathrm{~dB}$, which means that the correlation between Skip blocks and their side information blocks is strong and the Skip blocks can be replaced by the side information blocks. There is an exceptional case for Intra blocks and WZ blocks in Coastguard. As we can see, the APSNRs of Intra blocks and WZ blocks in Coastguard are higher than those of other videos. It is because the quality of SI is not bad due to Coastguard with well behaved motion.

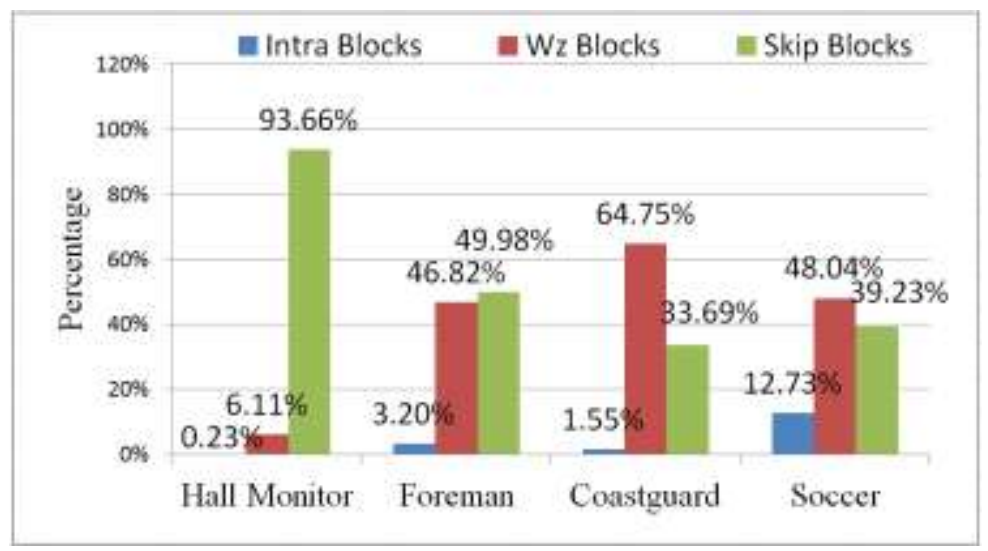

Figure 4. The Percentage of Each Mode in Test Videos

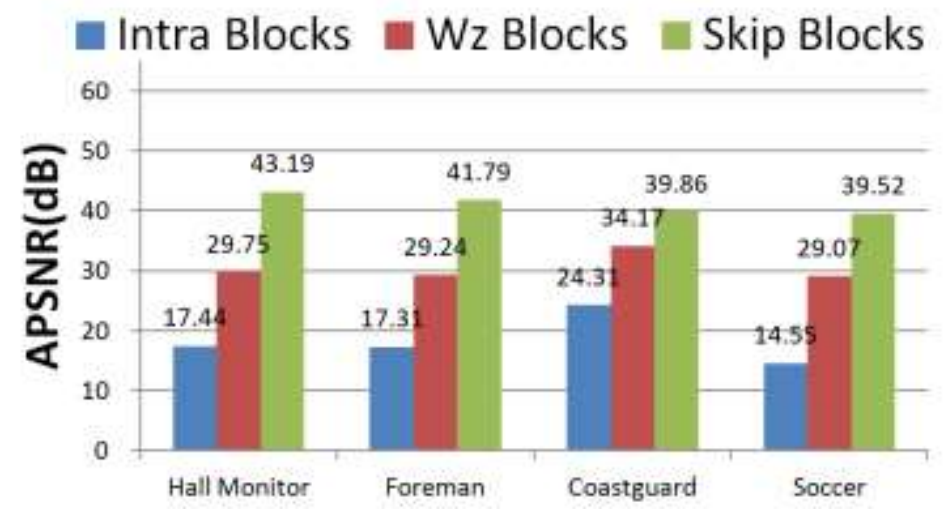

Figure 5. The APSNR of Each Kind of Block in Test Videos

Figure 6 shows the APSNR comparison of the Skip blocks with unsatisfactory side information blocks before and after using (5). It can be seen that the gains are up to about $2.8 \mathrm{~dB}, 2.1 \mathrm{~dB}, 1.2 \mathrm{~dB}$, and $1.4 \mathrm{~dB}$ in Hall Monitor, Foreman, Coastguard, and Soccer, respectively. The results show (5) is simple and helpful. 


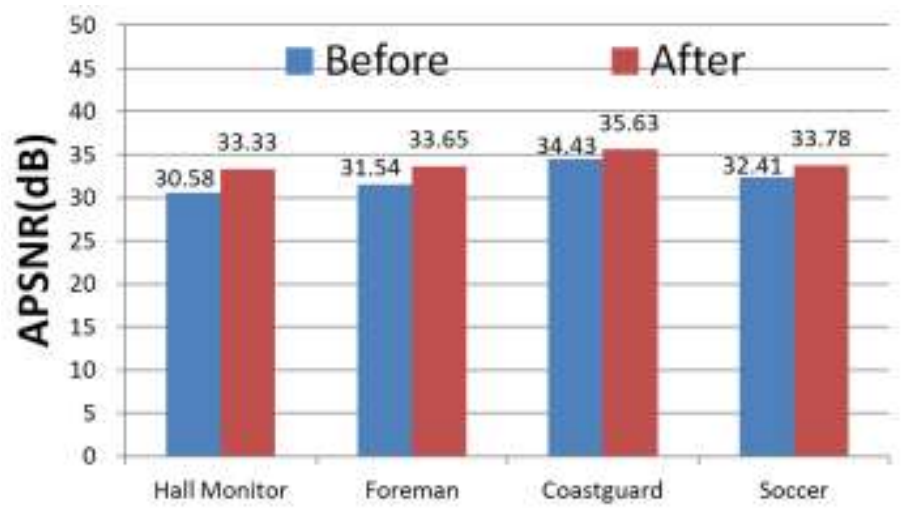

Figure 6. APSNR Comparison of Skip Blocks with Unsatisfactory SI Blocks Before and After Using (5)

In short, as we can see from the results of Figures 4-6, the proposed EBMD is very simple and effective in mode decision for the video with any degree of motion. Since the proposed EBMD is based on the hypothesis that $R_{q}^{\prime}=0$ accounts for $100 \%$, the satisfactory results also justify the hypothesis.

\subsection{RD Performance Comparisons}

Figure 7 shows the RD performance of the proposed EBMD-DRVC scheme for all the test videos, compared with DISCOVER [20] system and EFMD [9] system. DISCOVER is taken as the benchmark and the simulation results of DISCOVER come from [20]. EFMD is an encoder mode decision scheme which is implemented in WZ frame, while our EBMD-DRVC is implemented in residual frame. For convenience, only luminance components of these sequences are tested.

1. Compared with DISCOVER. DISCOVER is currently considered one of the best performing DVC systems which has been optimized in aspects e.g. the code rate control, correlation noise model, side information refinement and so on. The framework of DISCOVER is relatively complex. While the framework of EBMDDRVC shown in Figure 1 is very simple. Though the EBMD module is added at the encoder, it's very simple. Figure 7 shows that EBMD-DRVC performs better (the gain up to $1.4 \mathrm{~dB}$ on average) for Hall Monitor video which is explained that the Skip blocks are in the majority and help to improve the RD performance. For Coastguard, EBMD-DRVC provides a RD performance quite close to the one obtained by DISCOVER which is explained that WZ codec works well in Coastguard. As we can see from Figure 4 and Figure5 that the percentage and APSNR of WZ blocks in Coastguard are $64.75 \%$ and $34.17 \mathrm{~dB}$ respectively which mean WZ blocks are not only in the majority but also with satisfied SI, so the parity bits needed to correct the SI errors are not much which help to achieve good performance. For Foreman and Soccer video with high and complex motion, EBMD-DRVC presents a small RD performance gap (up to 0.4-1 dB) duo to the poor quality of SI. If the quality of SI can be improved by the side information refinement which is used in DISCOVER, the performance of EBMD-DRVC will be better but at the cost of increasing the complexity of the decoder. The simulation results show EBMD-DRVC can achieve good performances with simple framework, so it has a good practical use.

2. Compared with EFMD[9]. In [9], the paper proposes a simple encoder mode decision algorithm which depends on comparing both the temporal and the spatial $\mathrm{SAD}$ value. The temporal SAD is calculated between the co-located blocks in the adjacent frames and the spatial SAD is calculated between a block and its 
neighboring blocks using one of three equations. The best equation is chosen by heuristics. Fig, 7 shows that EBMD-DRVC outperforms EFMD with gains up to 2$6 \mathrm{~dB}$ in Hall Monitor video, $1-1.5 \mathrm{~dB}$ in Foreman video, about $1 \mathrm{~dB}$ in Coastguard video and about $2 \mathrm{~dB}$ in Soccer video. There are two reasons for such outcomes. One is that the absence of SI at the encoder in EFMD scheme causes inaccurate correlation estimation, so the mode decision in EFMD is not always effective. While in EBMD-DRVC scheme, the mode decision is based on the statistical characteristic of the decoder SI (seen in Section 3.2), even though there is no SI generated at the encoder, the mode decision is still effective. The other is Skip mode is introduced in EBMD-DRVC to save the transmitting data and the quality of co-located blocks in DSI can be improved by (5) which help to improve RD performance. Comparing the two, we can conclude that EBMD-DRVC and EFMD all belong to simple encoder mode decisions but EBMD-DRVC is more effective than EFMD.

3. Compared with H.264/AVC Intra. H.264/AVC Intra is the standard Intra coding solution. Figure 7 shows that EBMD-DRVC performs better for video sequences with low or well behaved motion, such as Hall Monitor and Coastguard. For Foreman and Soccer video sequences with high and complex motion, the RD performances of EBMD-DRVC are lower than that of H.264/AVC Intra codec which reveal if the percentage of Intra blocks in such videos account for more, the $\mathrm{RD}$ performance will be better.
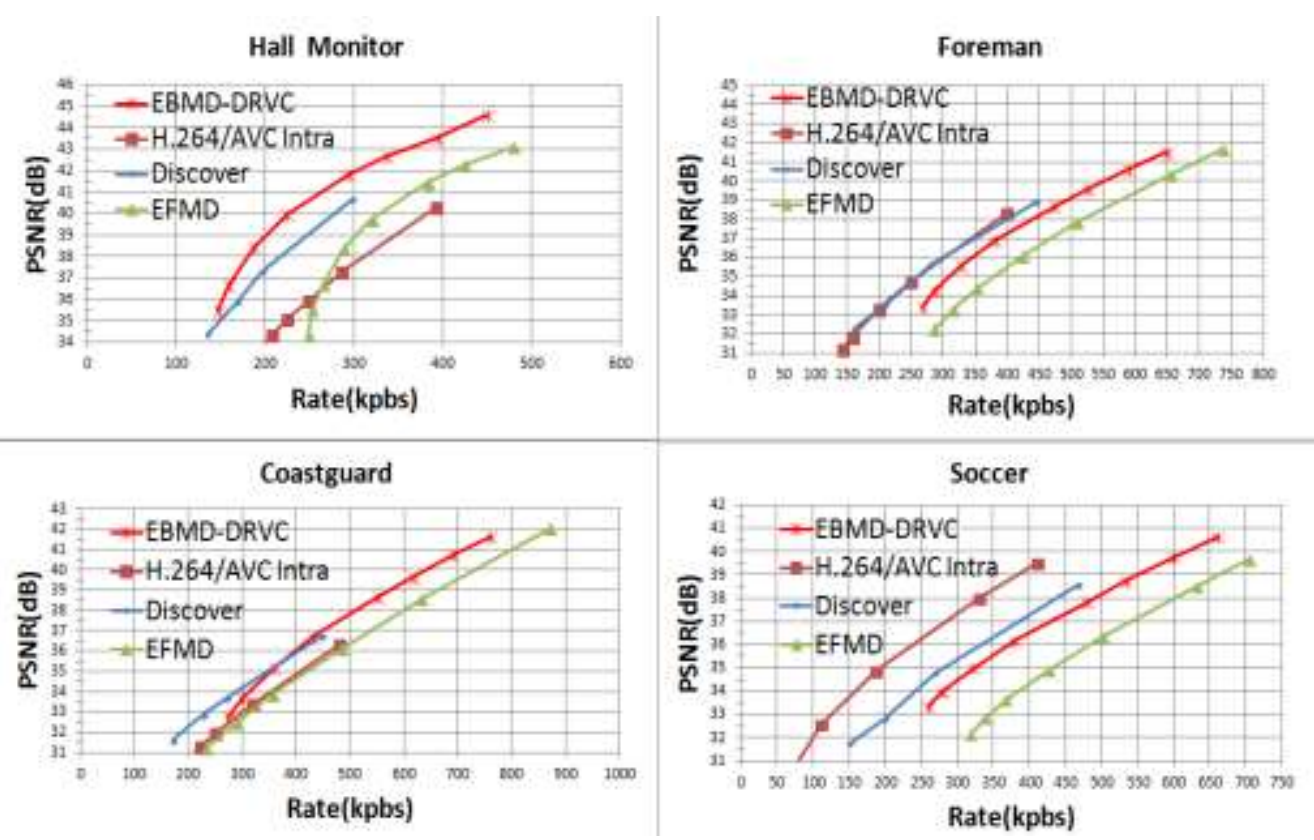

Figure 7. The RD Performance of EBMD-DRVC, DISCOVER and EFMD

\section{Conclusions}

The existing EBMD algorithms are unpractical in DVC system since they bring the computation load and complexity at the encoder. Aim to maintain the low-complexity nature of the encoder, we propose a simple and efficient EBMD-DRVC which only employs the value of the residual pixel at the encoder to decide the macro blocks into Intra mode, Skip mode and WZ mode. If the quality of the co-located blocks in DSI is unsatisfactory, the decoder can improve the quality of the reconstructed Skip blocks. Our metrics is very simple without any heavy computation and the threshold is fixed. These advantages guarantee a low-complexity encoder. The experiment results show EBMD- 
DRVC is efficient in mode decision and obtains good and better RD performance when compared with DISCOVER and EFMD, respectively. Due to having the low-complexity encoder, EBMD-DRVC is promising in practice. In the future work, we will study how to modify the mode decision criterion to increase the percentage of the Intra blocks in the videos with high and complex motion.

\section{Acknowledgments}

This work is supported by the Ministry of Industry and Information Technology of Things special fund([2014]351), the National Natural Science Foundation of China (No.61302055), the Specialized Research Fund for the Doctoral Program of Higher Education(No. 20120172120027), and the Project sponsored by SRF for ROCS, SEM.

\section{References}

[1] A. Aaron, R. Zhang and B. Girod, "Wyner-Ziv coding of motion video", The Thirty-Sixth Asilomar Conference on Signals Systems and Computers", Pacific Groove, CA, United states, (2002).

[2] A. Aaron, S. Rane, E. Setton and B. Girod, "Transform-domain wyner-ziv codec for video",Visual Communications and Image Processing 2004,San Jose, CA, United states, (2004).

[3] A. Aaron, D. Varodayan and B. Girod,"Wyner-Ziv residual coding of video",25th PCS: Picture Coding Symposium 2006, PCS2006,Beijing, China, (2006).

[4] W. Ji, P. Frossard and Y. Chen, "EXIT-Based Side Information Refinement in Wyner-Ziv Video Coding", IEEE Transactions on Circuits and Systems for Video Technology, vol. 24, no. 1, (2014), pp. 141-156.

[5] D. Y. Zhang, Y.-Y. Wu and M. X. Wan, "Improved side information generation algorithm for Wyner-Ziv video coding", The Journal of China Universities of Posts and Telecommunications, vol. 21, no. 1, (2014), pp. 109-115.

[6] Z.-H. Tang, X.-Y. Liang, T.-F. Qin and K. Chang, "Correlation noise modeling algorithm based on multiple probability distributions for distributed video coding", Acta Electronica Sinica, vol. 43, no. 2, (2015), pp. 365-370.

[7] H. Van Luong, L. L. Raket and S. Forchhammer, "Re-estimation of motion and reconstruction for distributed video coding", IEEE Transactions on Image Processing, vol. 23, no. 7, (2014), pp. 2804-2819.

[8] J.-C. Chiang, K.-L. Chen, C.-J. Chou, C.-M. Lee and W.-N. Lie, "Block-based distributed video coding with variable block modes", 2010 IEEE International Symposium on Circuits and Systems: Nano-Bio Circuit Fabrics and Systems, ISCAS 2010,,Paris, France, (2010).

[9] X. H. Van and B. Jeon, "Flexible complexity control solution for transform domain Wyner-Ziv video coding", IEEE Transactions on Broadcasting, vol. 58, no. 2, (2012), pp. 209-220.

[10] M. Tagliasacchi, A. Trapanese, S. Tubaro, J. Ascenso, C. Brites and F. Pereira, "Intra mode decision based on spatio-temporal cues in pixel domain Wyner-Ziv video coding", 2006 IEEE International Conference on Acoustics, Speech and Signal Processing, ICASSP 2006, Toulouse, France, (2006).

[11] L. Liu, D.-k. He, A. Jagmohan, L. Lu and E. J. Delp, "A low-complexity iterative mode selection algorithm Forwyner-Ziv video compression", 2008 15th IEEE International Conference on Image Processing,San Diego, CA, (2008).

[12] Y. Wang and C. Wu, "A block based Wyner-Ziv video codec", Image and Signal Processing (CISP), 2010 3rd International Congress on, Yantai, (2010).

[13] H.-P. Yang, H.-C. Hsieh, S.-H. Chang and S.-J. Chen, "An improved distributed video coding with lowcomplexity motion estimation at encoder", 28th IEEE International System on Chip Conference, SOCC 2015,Beijing, China, (2015).

[14] V. Kumar and S. Sengupta, "Decoder driven multi resolution side information refinements and mode decisions for improved rate-distortion performance in distributed video coding",2011 12th IEEE International Conference on Multimedia and Expo, ICME 2011,Barcelona, Spain, (2011).

[15] J. Slowack, S. Mys, J. Korupa, N. Deligiannis, P. Lambert, A. Munteanu and R. Van De Walle, "Ratedistortion driven decoder-side bitplane mode decision for distributed video coding", Signal Processing: Image Communication, vol. 25, no. 9, (2010), pp. 660-673.

[16] J. Skorupa, J. Slowack, S. Mys, N. Deligiannis, J. De Cock, P. Lambert, C. Grecos, A. Munteanu and R. Van de Walle,"Efficient low-delay distributed video coding", IEEE Transactions on Circuits and Systems for Video Technology, vol. 22, no. 4, (2012), pp. 530-544.

[17] G. Qiu, S. Gao and G. Tu, "Low-complexity low-delay distributed video coding", 2013 18th International Conference on Digital Signal Processing, DSP 2013,Santorini, Greece, (2013).

[18] J. Ascenso, C. Brites and F. Pereira, "Improving frame interpolation with spatial motion smoothing for pixel domain distributed video coding", 5th EURASIP Conference on Speech and Image Processing, Multimedia Communications and Services, Fira, (2005).

[19] http://www.img.lx.it.pt/ discover/rd_qcif_15_gop2.html. 

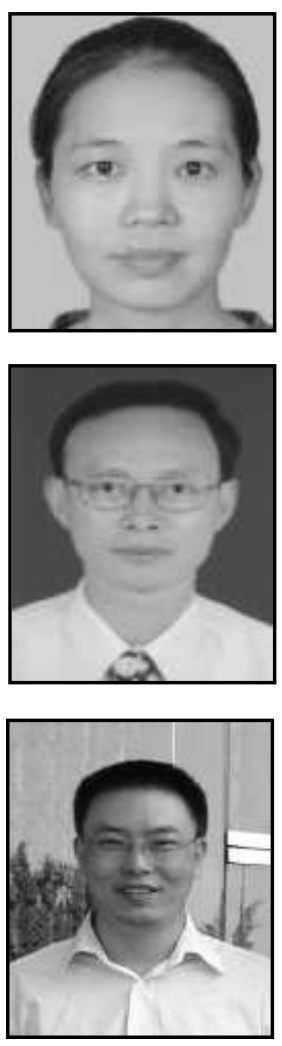

\section{Authors}

Chunyun Hu, received her Master degree in School of Electronic and Information Engineering South China University of Technology. She is currently a lecturer at College of Electronic and Engineering in South China Agricultural University. Her research interests include DVC, the routing problems in WSNs.

Binjie Hu, is the Doctoral Advisor in school of Electronic and Information Engineering South China University of Technology. His research interests include Wireless sensor networks and cognitive radio technology, radio frequency identification (RFID) and Internet of things technology.

Yunhui Xiong, is currently an assistant professor of the College of Science in South China University of Technology. His research interests are computer graphics, shape analysis, image editing and video coding. 
International Journal of Signal Processing, Image Processing and Pattern Recognition Vol. 10, No. 7 (2017) 